\title{
Validation of a new method for the endoscopic measurement of post-bariatric gastric outlet using a standard guidewire: an observer agreement study
}

\author{
Luiz Gustavo de Quadros 1,2,3* , Manoel dos Passos Galvão Neto ${ }^{3,4}$, Josemberg Marins Campos ${ }^{3,5}$, \\ Roberto Luiz Kaiser Junior ${ }^{1}$, Eduardo Grecco ${ }^{2,3}$, Mario Flamini Junior ${ }^{1}$, Marcelo Falcao de Santana ${ }^{3,5}$, \\ Idiberto Jose Zotarelli Filho ${ }^{6}$ and Adriano Augusto Tomas Vasconcelos Almeida Alexandre ${ }^{5}$
}

\begin{abstract}
Background and aims: Between 10 and 20\% of all patients undergoing bariatric surgery procedures regain weight secondary to a gastrojejunostomy enlargement. The aim of this study was to validate the interobserver agreement while measuring gastric outlet diameters using a new standard guidewire.

Methods: We selected thirty-five videos of consecutive endoscopic procedures on patients undergoing esophagogastroduodenoscopy after a Roux-en-Y gastric bypass procedure. All videos were evaluated by four raters: two expert endoscopists and two trainees. We excluded videos having a slipped Fobi ring or a strictured gastric outlet. Anastomosis diameter was measured using a novel device with standardized markings on a guidewire (Hydra jagwire, Boston Scientific, Natick. MA) as well as the current gold standard defined as a calibrated endoscopic measuring instrument (Olympus America, Center Valley, PA).
\end{abstract}

Results: We obtained 272 measurements of the gastric outlet. Overall agreement measured through intra-class correlation coefficients for the gold standard was $0.84(p<0.01)$ and $0.83(p<0.01)$ for the new guidewire. Agreement among experts was $0.699(p<0.01)$, while among trainees it was $0.822(p<0.01)$.

Conclusion: The new guidewire demonstrated a high degree of observer reliability, also presenting similar results between expert endoscopists and trainees.

Keywords: Gastric outlet, Observer agreement, Measurement reliability, Weight regain, Weight recidivism, Upper endoscopy

\section{Background}

Although bariatric surgery procedures effectively treat morbid obesity and reduce rates of long-term obesity-related complications [1, 2], weight regain might occur in up to $20 \%$ of all patients. Among a multitude of possible factors associated with weight regain, gastric outlet dilation occurs when its diameter exceeds $14-20 \mathrm{~mm}$, ultimately leading patients

\footnotetext{
*Correspondence: gustavo_quadros@hotmail.com

1 Department of Endoscopy and Bariatric Surgery, Kaiser Clinic and Day Hospital, São José do Rio Preto, SP 15015-110, Brazil

Full list of author information is available at the end of the article
}

back to obesity [3]. Although outlet measurement is an essential step in defining a therapeutic plan, to our knowledge there are no previous studies evaluating the reliability associated with different measurement alternatives.

A number of therapeutic options have been developed to reduce the anastomotic diameter, including endoscopic suturing devices and gastrojejunostomy [4-6]. Abnormal anatomical findings are found in $71.2 \%$ of all patients, $58.9 \%$ of which have a dilated anastomosis, $28.8 \%$ present a dilated pouch, and $12.3 \%$ with both conditions [7].

Currently, there seems to be no consensus on how to best measure anastomosis diameter. Devices designed to measure 
these dimensions frequently differ in both units and mechanisms, including balloons, clamps and spacers. While most authors use gaging through a grasper-type forceps connected to the endoscope working channel, $[8,9]$ no consensus exists on which differences might exist when different measurement tools might be used. In addition, grasper-type forceps are both expensive and hard to find in developing countries.

The objective of this study was therefore to validate a novel, efficient and inexpensive method to measure gastric outlet diameter, evaluating its agreement reliability.

\section{Methods}

\section{Ethics}

This prospective study was approved by the Institutional Review Board of the Hospital Beneficiencia of São José do Rio Preto. Informed consent was obtained by all participants prior to the implementation of any study protocol.

\section{Cases and measurement}

All patients were consecutively recruited through the Digestive Endoscopy Service of the Kaiser Clinic, Brazil. Patients were included if they had undergone gastric bypass surgery with or without a Fobi band. The endoscopic procedure was conducted as a routine exam which is part of our postoperative protocol and regardless of existing symptoms. Patients were excluded if they had been found to have a gastrojejunostomy stenosis or a slipped ring.

After sedation, an upper endoscopic procedure was conducted by an expert endoscopist using an Olympus CV-180 or 160 endoscope (Olympus, Tokyo, Japan). All measurements were recorded in high-definition. All patient identifiers were stripped from the video in order to ensure patient confidentiality.

The anastomosis diameter was initially measured through an articulated device for anastomosis scouting with distal markings at every $0.2 \mathrm{~mm}$ by $0.2 \mathrm{~mm}$ (Fig. 1 ).

A second measurement was then conducted with the experimental guidewire, custom manufactured from a Wire Guide Hydra Jagwire (Boston Scientific) with a hydrophilic flexible tip painted in black and additional black stripes every $0.5 \mathrm{~mm}$.

A total of 35 videos from endoscopic exams conducted on 35 patients were rated by four raters, two expert endoscopists and two clinicians in training.

\section{Data analysis}

We started the analysis with a graphical exploration of the sample data, evaluating means and standard deviation for numeric variables, as well as frequencies and percentages for categorical variables. Numeric variables were also evaluated for their distribution and extreme values. The association across ratings was further evaluated through a correlation graphic. Intra-class correlation coefficients

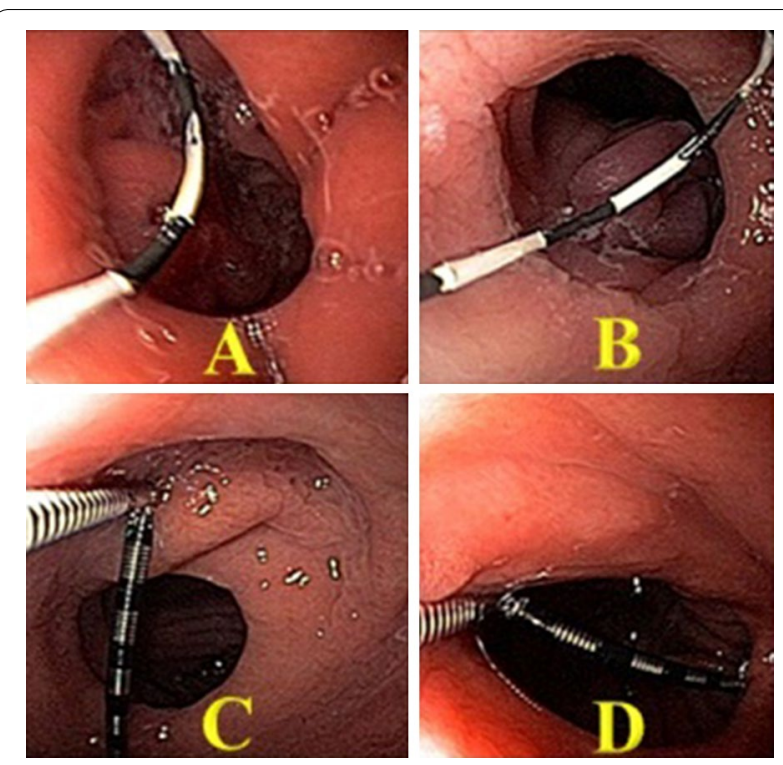

Fig. 1 Diameter measurement of the gastrojejunal anastomosis through the guidewire $(\mathbf{A}, \mathbf{B})$ and gold standard method $(\mathbf{C}, \mathbf{D})$

were used to evaluate the inter-rater reliability of ratings between novice and expert raters, standard and guidewire, and combinations among the previous categories. Finally, a Bland-Altman plot was used to assess the agreement reliability comparing experts and novice raters.

\section{Results}

No injuries or complications occurred during any of the measurements. Patients' average age was $38.46 \pm 10.61$, with $77.14 \%(n=27)$ of them being women.

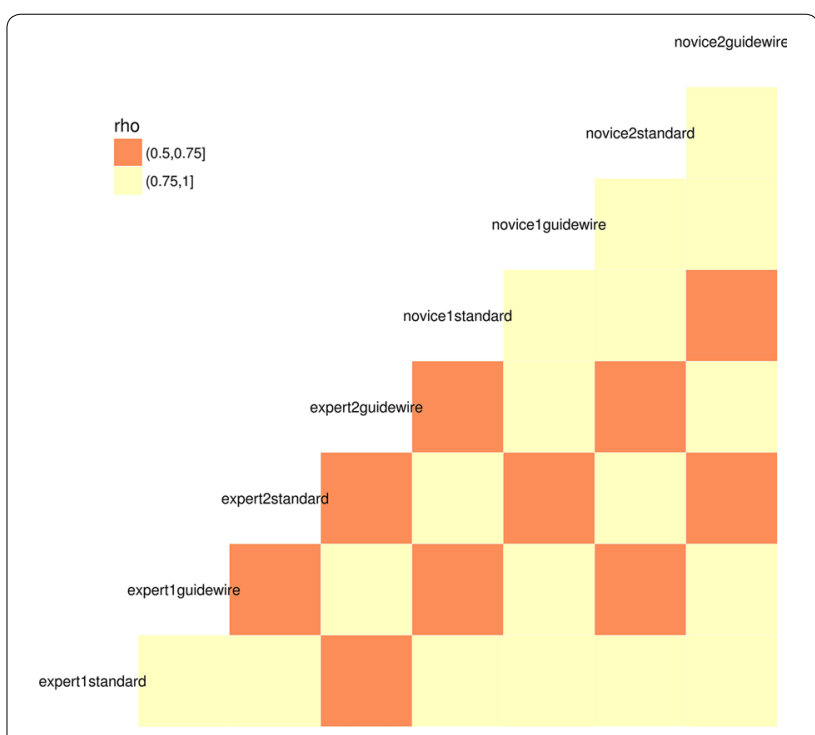

Fig. 2 Correlation plot for measurements across raters 
When visually inspecting the correlation among different raters, there was a higher correlation in ratings within experts as well as within novice raters. In addition, the agreement using the guidewire and the gold standard made by the same rater were higher than across other raters (Figs. 2, 3).

Overall agreement measured through intra-class correlation coefficients for the gold standard was 0.84 $(\mathrm{p}<0.01)$ and $0.83(\mathrm{p}<0.01)$ for the new guidewire. Agreement among experts was $0.699(\mathrm{p}<0.01)$, while among trainees it was $0.822(\mathrm{p}<0.01)$. When evaluating subgroups, intra-class correlation coefficients for trainees evaluating the gold standard was 0.877 $(\mathrm{p}<0.01)$, trainees evaluating the new guidewire was $0.865(\mathrm{p}<0.01)$, experts evaluating the gold standard was $0.795(\mathrm{p}<0.01)$, and experts evaluating the guidewire was $0.843(\mathrm{p}<0.001)$. These high rates of agreement are confirmed through the Bland-Altman plot comparing novice and expert raters (Fig. 4). This figure displays the rates of agreement among observers falling within an

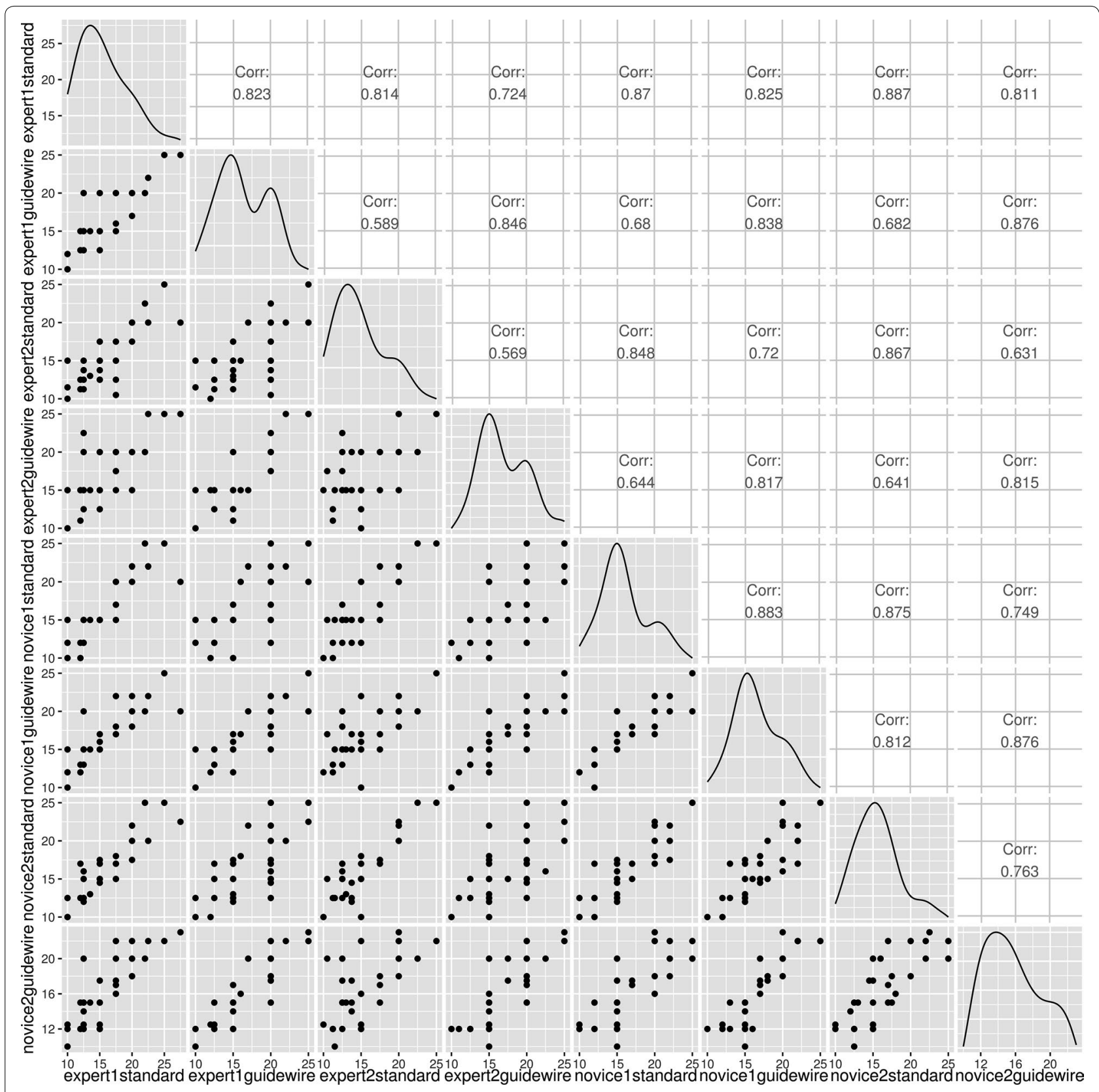

Fig. 3 Association among rater measurements 


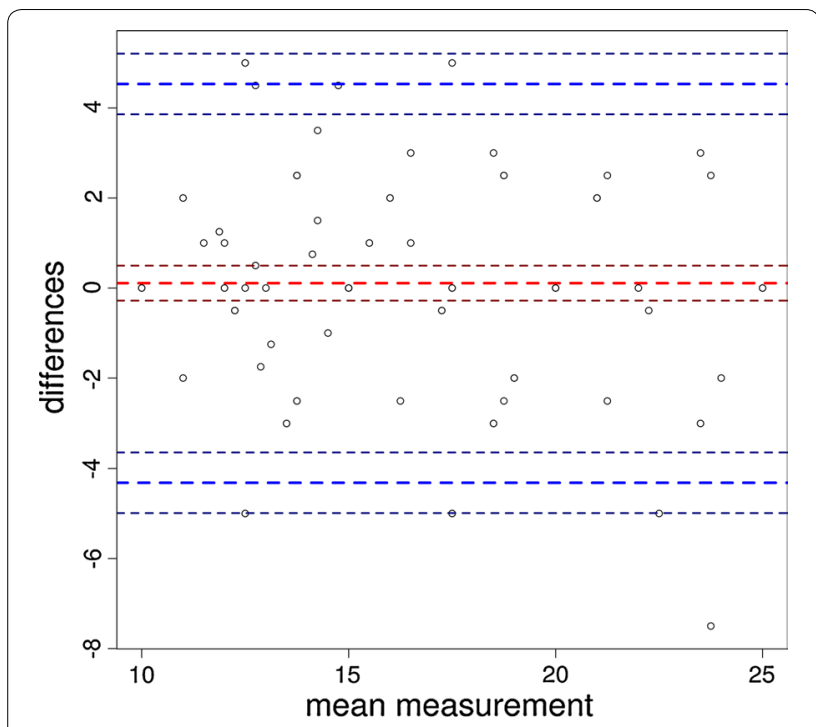

Fig. 4 Bland-Altman plot comparing expert and novice raters

acceptable range (within the two horizontal lines). The horizontal lines represent the mean difference in agreement plus or minus 1.96 times the standard deviation of the differences.

\section{Discussion}

We found that our novel guidewire instrument has a high agreement reliability in comparison with the gold standard Olympus calibration device. This high agreement reliability is maintained for both expert and novice raters. In addition, no patients demonstrated any complications during the measurement procedures in this sample. These results are important in that the gold standard Olympus device is not only expensive, but of difficult acquisition in developing countries. Our novel device should therefore increase access to this measurement procedure.

In an attempt to avoid weight regain, reducing the diameter of a dilated anastomosis may lead to a reduction of up to $23 \%$ in weight [10]. Most studies will recommend a $20 \mathrm{~mm}$ diameter, although diameters as small as $12 \mathrm{~mm}$ have been proposed [11]. Despite this growing body of evidence regarding the importance of the anastomosis diameter, the literature can be challenged if the measurement process has not been properly validated.

Our results are clinically relevant to clinical practice since approximately $20-30 \%$ of all bariatric patients regain weight, many of these being addressed through an endoscopic procedure $[12,13]$. The precision in these procedures is essential, in that an anastomosis with a diameter smaller than $5 \mathrm{~mm}$ would prevent patients from digesting liquids, a diameter smaller than $10 \mathrm{~mm}$ would prevent patients from digesting solid food, while diameters greater than $14 \mathrm{~mm}$ are associated with weight regain $[11,14]$. The margin for error is therefore small, thus requiring not only measurement precision but also a high degree of reliability across measurements. In addition, it is important for clinicians to communicate with patients so that they can be informed about the current status of their procedure, but also participate in decisions that will affect their ability to eat and drink [15, 16]. In addition, this novel device increases access to this measurement method in countries where the traditional device is either difficult to find or expensive to incorporate into clinical practice.

Although we propose a new and inexpensive and reliable method for measuring dilation, our study does have limitations. First, we evaluated the reliability of videos rather than conducted new endoscopic procedures. While conducting procedures on the same patient by different endoscopists would enhance our methodology, this methodology would pose risks and discomfort to our patients, and was therefore avoided. Second, our sample was small and restricted to a single center. Future studies should therefore further validate our results with more representative samples.

In summary, our study presents a novel, simple, safe, accurate and inexpensive method to measure the outlet diameter. These findings are relevant in that the gold standard Olympus calibration device is not only of difficult access in a number of countries, but also expensive. Given that we have demonstrated equivalent measurement reliability between the Olympus device and our new guidewire, both with experienced endoscopists and trainees, we recommend its use in clinical and research practice.

\section{Authors' contributions}

LGQ participated in conception and design of the study, analysis and interpretation of data, drafting of the manuscript and final approval of the manuscript. MGN Conception and design of the study, analysis and interpretation of data, reviewing and final approval of the manuscript. JMC carried out analysis and interpretation of data, reviewing and final approval of the manuscript. RKJ and IZF carried out analysis and interpretation of data and drafting of the manuscript. EG participated in conception and design of the study and reviewing of the manuscript. MFJ carried out analysis and interpretation of data and reviewing of the manuscript. MFS participated in drafting of the manuscript. AAA participated in drafting and reviewing of the manuscript. All authors read and approved the final manuscript.

\section{Author details \\ ${ }^{1}$ Department of Endoscopy and Bariatric Surgery, Kaiser Clinic and Day Hospital, São José do Rio Preto, SP 15015-110, Brazil. ${ }^{2}$ Department of Diges- tive Surgery, School of Medicine of ABC, Santo Andre, SP 09080-650, Brazil. \\ ${ }^{3}$ Brazilian Bariatric Endoscopy International Group, São Paulo, Brazil. ${ }^{4}$ Gastro Obeso Center, São Paulo, SP 01308-000, Brazil. ${ }^{5}$ Department of Surgery, Federal University of Pernambuco (UFPE), Recife, PE 50670-901, Brazil. ${ }^{6}$ State University of Sao Paulo-Unesp-Ibilce, Rua Cristovão Colombo 2265, Sao Jose do Rio Preto, SP 15054-000, Brazil.}

\section{Acknowledgements}

We thank the logistical support provided by the Kaiser Clinic as well as Larissa Souza, MD, Dr. Fernando Tadeu Vannucci Coimbra and Dr. Ivan Enokibara da Silva from the Gastro Imagem, Sao Jose do Rio Preto, Brazil for their support. 


\section{Competing interests}

The authors declare that they have no competing interests.

\section{Availability of data and materials}

The datasets during and/or analyzed during the current study available from the corresponding author on reasonable request.

\section{Ethics approval and consent to participate}

This study was reviewed and approved by the Institutional Review Board of the Hospital Beneficiencia of São José do Rio Preto and all participating patients provided written informed consent.

Received: 20 October 2016 Accepted: 16 December 2016

Published online: 03 January 2017

\section{References}

1. da Costa RCNC, Yamaguchi N, Santo MA, Riccioppo D, Pinto-Junior PE. Outcomes on quality of life, weight loss, and comorbidities after Roux-enY gastric bypass. Arq Gastroenterol. 2014;51:165-70.

2. Sjöström L, Lindroos A-K, Peltonen M, Torgerson J, Bouchard C, Carlsson $B$, et al. Lifestyle, diabetes, and cardiovascular risk factors 10 years after bariatric surgery. N Engl J. 2004;351:2683-93.

3. Wang B, Levine MS, Rubesin SE, Williams NN, Dumon K, Raper S. Utility of barium studies for patients with recurrent weight gain after Roux-en-Y gastric bypass. Clin Radiol. 2015;70:67-73.

4. Paluszkiewicz R, Kalinowski P, Wróblewski T, Bartoszewicz Z, BiałobrzeskaPaluszkiewicz J, Ziarkiewicz-Wróblewska B, et al. Prospective randomized clinical trial of laparoscopic sleeve gastrectomy versus open Roux-en-Y gastric bypass for the management of patients with morbid obesity. Wideochir Inne Tech Maloinwazyjne. 2012;7:225-32.

5. Kumar N. Endoscopic therapy for weight loss: gastroplasty, duodenal sleeves, intragastric balloons, and aspiration. World J Gastrointest Endosc. 2015;7:847-59.
6. Ryou M, Thompson CC. Current status of endoluminal bariatric procedures for primary and revision indications. Gastrointest Endosc Clin N Am. 2011:21:315-33.

7. Yimcharoen $\mathrm{P}$, Heneghan HM, Singh M, Brethauer S, Schauer P, Rogula T, et al. Endoscopic findings and outcomes of revisional procedures for patients with weight recidivism after gastric bypass. Surg Endosc. 2011:25:3345-52

8. Halperin F, Ding S-A, Simonson DC, Panosian J, Goebel-Fabbri A, Wewalka $M$, et al. Roux-en-Y gastric bypass surgery or lifestyle with intensive medical management in patients with type 2 diabetes: feasibility and 1-year results of a randomized clinical trial. JAMA Surg. 2014;149:716-26.

9. Lehmann A, Bobowicz M, Lech P, Orłowski M, Siczewski W, Pawlak M, et al. Comparison of percentage excess weight loss after laparoscopic sleeve gastrectomy and laparoscopic adjustable gastric banding. Wideochir Inne Tech Maloinwazyjne. 2014;9:351-6.

10. Sjöström L. Review of the key results from the Swedish Obese Subjects (SOS) trial-a prospective controlled intervention study of bariatric surgery. J Intern Med. 2013:273:219-34.

11. Levine MS, Carucci LR. Imaging of bariatric surgery: normal anatomy and postoperative complications. Radiology. 2014;270:327-41.

12. Pajecki D, Dalcanalle L, De Oliveira CPMS, Zilberstein B, Halpern A, Garrido AB Jr, et al. Follow-up of roux-en-y gastric bypass patients at 5 or more years postoperatively. Obes Surg. 2007;17:601-7.

13. Bastos ECL, Barbosa EMWG, Soriano GMS, dos Santos EA, Vasconcelos SML. Determinants of weight regain after bariatric surgery. Arq Bras Cir Dig. 2013;26:26-32.

14. Huang CS, Forse RA, Jacobson BC, Farraye FA. Endoscopic findings and their clinical correlations in patients with symptoms after gastric bypass surgery. Gastrointest Endosc. 2003;58:859-66.

15. Arterburn DE, Courcoulas AP. Bariatric surgery for obesity and metabolic conditions in adults. BMJ. 2014;349:g3961.

16. Arterburn DE, Westbrook EO, Bogart TA, Sepucha KR, Bock SN, Weppner WG. Randomized trial of a video-based patient decision aid for bariatric surgery. Obesity. 2011;19:1669-75.

\section{Submit your next manuscript to BioMed Central and we will help you at every step:}

- We accept pre-submission inquiries

- Our selector tool helps you to find the most relevant journal

- We provide round the clock customer support

- Convenient online submission

- Thorough peer review

- Inclusion in PubMed and all major indexing services

- Maximum visibility for your research

Submit your manuscript at www.biomedcentral com/submit
() Biomed Central 